\title{
Uma proposta teórico-metodológica para elaboração de modelos teóricos
}

\section{A theoretical-methodological proposal for the elaboration of theoretical models}

\author{
Breno Augusto Bormann de Souza Filho ${ }^{1 *}$ (D), Cláudio José Struchiner ${ }^{1}$ (D) \\ 'Departamento de Epidemiologia, Escola Nacional de Saúde Pública - ENSP, Rio de Janeiro (RJ), Brasil.
}

Como citar: Souza Filho BAB, Struchiner CJ. Uma proposta teórico-metodológica para elaboração de modelos teóricos. Cad Saúde Colet, 2021;29(1):86-97. https://doi.org/10.1590/1414-462X202129010180

\section{Resumo}

Introdução: Trabalhos que abordem a importância da utilização dos Modelos Teóricos nas pesquisas são escassos, bem como instruções para sua elaboração. Objetivo: Apresentar uma proposta teóricometodológica para construção de Modelos Teóricos. Método: Trata-se de uma revisão narrativa, cujo processo de coleta do material foi realizado de forma não sistemática, eleitos exclusivamente e baseados na linha de argumentação e reflexão que os autores pretendem submeter à apreciação e ao debate público. A coleta foi realizada de julho de 2018 a março de 2019, nos idiomas português, espanhol e inglês. Resultados: A importância da utilização dos Modelos Teóricos foi apresentada, bem como um passo a passo de sete etapas para sua elaboração: Identificação e Delimitação do Objeto de Estudo (Etapa 1); Resgate Cognitivo e Tempestade de Ideias (Etapa 2); Representação do Modelo Teórico (Etapa 3); Revisão da Literatura sobre o Tema (Etapa 4); Estruturação do Modelo Teórico (Etapa 5); Submissão do Modelo Teórico a Especialistas (Etapa 6); até a Reestruturação e Finalização do Modelo Teórico (Etapa 7). Conclusão: Espera-se que este artigo levante discussões e reflexões sobre os métodos atuais que permeiam o fazer científico e fomentem a utilização dos Modelos Teóricos como base das pesquisas científicas.

Palavras-chave: modelos teóricos; conhecimento; saúde pública.

\begin{abstract}
Introduction: Few papers address the importance of using Theoretical Models in research, as well as instructions for their elaboration. Objective: To present a theoretical-methodological proposal for the construction of theoretical models. Method: This is a narrative review, whose material collection was carried out in a non-systematic way, with papers being elected based exclusively on the line of argument and reflection the authors intended to submit to public appreciation and debate. Collection was carried out from July 2018 to March 2019, in Portuguese, Spanish and English. Results: The importance of using Theoretical Models was presented, as well as a 7-step process for their elaboration: Identification and Delimitation of the Study Object (Step 1); Cognitive Rescue and Storm of Ideas (Step 2); Representation of the Theoretical Model (Step 3); Literature Review on the Theme (Step 4); Drawing up of the Theoretical Model (Step5); Submission of the Theoretical Model to Specialists (Step 6); and Restructuring and Completion of the Theoretical Model (Step 7). Conclusion: It is hoped that this article will raise discussions and reflection on the current methods that permeate scientific creation and encourage the use of Theoretical Models as the basis of scientific research.
\end{abstract}

Keywords: theoretical models; knowledge; public health.

Este é um artigo publicado em acesso aberto (Open Access) sob a licença Creative Commons Attribution, que permite uso, distribuição e reprodução em qualquer meio, sem restrições desde que o trabalho original seja corretamente citado. 


\section{INTRODUÇÃO}

Embora a literatura apresente que modelos teóricos devam ser incorporados e servir como norteadores para todas as etapas das pesquisas científicas, atualmente sua representação e explicação são relativamente obscuras entre a vasta literatura disponível ${ }^{1,2}$. A maioria dos artigos publicados não apresenta explicações robustas sobre o que, como, ou quais são as estruturas teóricas e conceituais abordadas e, quando informadas, são mencionadas minimamente e/ou frequentemente como termos em um glossário ${ }^{1,3,4}$.

Como questões influentes desse cenário, pode-se citar a alta produtividade exigida por organizações de fomento, aliada à maior valorização de estudos que apresentam resultados estatisticamente significativos ${ }^{5-8}$ e cujo desenho possibilite achados com maiores níveis de evidência ${ }^{6,9}$. Nesse sentido, evidencia-se uma crescente desvalorização das bases teóricas na elaboração das pesquisas ${ }^{10,11}$.

Outrossim, ainda são escassos na literatura trabalhos que abordem a importância da utilização dos modelos teóricos nas pesquisas científicas, bem como instruções para sua elaboração'. Tal escassez, pode servir como uma barreira limitante para a elaboração e utilização dos Modelos Teóricos pelos pesquisadores nos estudos científicos.

Portanto, o objetivo deste artigo é apresentar uma proposta teórico-metodológica para construção de modelos teóricos, na forma de um instrutivo para pesquisadores, de modo que evidencie um passo a passo para elaboração de modelos teóricos.

\section{MÉTODO}

O presente estudo consiste em uma revisão de literatura do tipo narrativa, a qual intenta realizar uma síntese qualitativa em que o autor possui liberdade para interpretar e analisar criticamente achados na literatura, possibilitando a descrição e discussão do "estado da arte" sobre o tema ${ }^{12}$. Além disso, esse tipo de estudo apresenta importante papel na educação continuada, pois permite ao leitor atualizar-se sobre um tema específico em tempo reduzido ${ }^{12}$.

O processo de coleta do material foi realizado de forma não sistemática no período de julho de 2018 a março de 2019. Foram pesquisadas bases de dados científicas, tais como: Medline (Medical Literature Analysis and Retrieval System Online) via PUBMED; SciELO (Scientific Eletronic Library Online) e LILACS (Literatura Latino-Americana e do Caribe de Informação em Ciências da Saúde) via BVS (Biblioteca Virtual em Saúde); SCOPUS e Web of Science via portal de periódicos CAPES. Não houve restrições relacionadas ao período das publicações. Os idiomas utilizados para leitura dos artigos foram: espanhol, inglês e português.

A busca bibliográfica foi orientada a partir da Teoria da Aprendizagem Significativa ${ }^{13}$. Dessa forma, foram utilizados os seguintes descritores ou termos relacionados via DeCS (Descritores em Ciências da Saúde) e seus respectivos correspondentes pelo MeSH (Medical Subject Headings): teoria / theory; modelos teóricos / models, theoretical; constructo teórico / theoretical construct; constructo / construct; quadro teórico / theoretical framework; quadro conceitual / conceptual framework; Aprendizagem / Learning; Pesquisa / Research. Os termos foram combinados utilizando o operador booleano "OR" $\mathrm{e}$ "AND" para aumentar sensibilidade e especificidade dos achados, respectivamente.

Além disso, o banco de dados foi complementado com materiais indicados por especialistas na temática e aqueles relacionados à Teoria da Aprendizagem Significativa.

A seleção e extração de dados foi realizada pelo primeiro autor (BABSF). Os materiais foram lidos na íntegra, categorizados e analisados com intuito de corroborar e fortalecer a linha de raciocínio e plausibilidade científica apresentada pelos autores acerca do desenvolvimento da proposta metodológica para construção de Modelos Teóricos.

Por fim, por se tratar de uma revisão narrativa, a síntese dos resultados foi realizada por meio de descrição narrativa gerada através de interpretações qualitativas e reflexões dos autores sobre os tópicos relacionados ao desenvolvimento de modelos teóricos, não sendo assim, realizada avaliação da qualidade dos estudos incluídos nesta revisão. 


\section{RESULTADOSE DISCUSSÃO}

Com o intuito de descrever um passo a passo para elaboração de Modelos Teóricos, o presente trabalho iniciará definindo e diferenciando a Teoria de Modelos Teóricos.

\section{O que são Teorias?}

Teoria é caracterizada como um conjunto de conceitos, definições e proposições interrelacionadas que apresentam uma visão sistemática de eventos ou situações, especificando as relações entre as variáveis observadas, a fim de explicar e prever os eventos ou situações em questão ${ }^{14}$. A teoria também pode ser explicada como o conhecimento organizado que é aplicável a uma grande variedade de circunstâncias de modo a analisar, prever ou explicar a natureza ou comportamento de um fenômeno ${ }^{15}$.

Nesse sentido, é observável que a teoria pode e deve ser usada como base para prática, uma vez que autores defendem que a ação valida a teoria explicitada e/ou compreendida nos modelos teóricos. Entretanto, é importante evidenciar que as teorias podem ser postuladas sem a necessidade de uma demonstração empírica ${ }^{15}$.

O processo teórico tem por objetivo a interpretação de parte da realidade. Desse modo, segundo Bunge ${ }^{16}$, a força da teorização é resultado da capacidade das Teorias Gerais - que por si só não dizem respeito a nada - de, ao serem inseridas por objetos conceituais (ObjetosModelo), produzirem representações da realidade, isto é, Modelos Teóricos.

\section{O que são Modelos Teóricos?}

Modelo teórico é compreendido como um sistema hipotético-dedutivo representante da realidade, que pode gerar proposições a partir de proposições iniciais, ou seja, é possível realizar previsões a partir deles e simular o real ${ }^{16,17}$.

Para tanto, todo modelo deve definir e demonstrar os mecanismos que sustentam suas relações. Esses mecanismos, hipotéticos ou escondidos, podem estar ou não acessíveis à percepção, sendo sumariamente inferências teóricas passíveis de confirmações empíricas. É evidenciada, dessa forma, a característica de falibilidade e refutabilidade dos modelos teóricos e, por isso, sua constante transformação e adaptação no horizonte temporal da ciência.

Nesse contexto, os modelos teóricos são estruturas elaboradas quando há necessidade de encontrar relações entre teoria e dados empíricos. Sendo, então, os Modelos uma peça fundamental e intermediária do fazer científico: conceitos e medidas.

Diferente da realidade, as teorias apresentam-se como meras abstrações da mente humana. Assim, não têm valor se observadas por si só, desatreladas da realidade. Por outro lado, os dados empíricos, apesar de serem medidas muitas vezes consideradas mais próximas da realidade, não podem gerar conhecimento por si só, uma vez que são compreendidos como símbolos, o que requer algo que os interprete. A partir desta aparente dicotomia entre teórico e empírico, Bunge ${ }^{16}$ introduz os Modelos Teóricos como instância mediadora.

Desta forma, ressalta-se a impossibilidade de testar Teorias Gerais, dado que, apesar de estabelecerem relações dedutivas, não têm como foco o universo empírico (realidade). O que também se aplica aos Objetos Modelo; não há importância em definir seu status de verdadeiro ou falso, pois, em se tratando de decisões arbitrárias em função das decisões do pesquisador, circunscrito num universo temporal, adquirem status apenas convencional. Entretanto, os Modelos Teóricos, por serem produzidos a partir de Teorias Gerais com o uso de diferentes Objetos Modelos em interação, podem ser testados empiricamente e, assim, gerar problemas e serem refutados ${ }^{16}$.

Por fim, para Bunge ${ }^{16}$, os modelos teóricos são considerados a essência do trabalho científico.

Entretanto, para alguns autores, a nomenclatura não é o mais importante e sim a compreensão de como a teoria ou modelo teórico têm sido utilizados por pesquisadores para sustentar o estudo ${ }^{18,19}$, compreendendo que o modelo teórico adotado deve ser claramente 
identificado e explicitado em qualquer pesquisa e publicação científica, de forma a orientar tanto o pesquisador e sua equipe, quanto o leitor ${ }^{20}$.

\section{Formas e Funções dos Modelos Teóricos para a Ciência}

A grande utilidade dos Modelos Teóricos está em possibilitar a simplificação de uma realidade através da delimitação de uma questão específica ${ }^{21}$.

Dessa forma, é possível sintetizar que o trabalho científico consiste, em grande parte, na construção de modelos que sirvam como representação dos fenômenos estudados, integrados em teorias e que apresentem a capacidade de resolver problemas ${ }^{22}$.

Os modelos teóricos podem ser representados de várias maneiras. Comumente a forma textual é a mais utilizada pelos pesquisadores. Entretanto a literatura aponta que o Modelo pode ser mais bem compreendido se representado através de símbolos, desenhos, mapas conceituais e/ou diagramas, os quais demonstrem as bases teóricas das relações que o cientista ou pesquisador pretende extrair e/ou observar da natureza ou da sociedade ${ }^{21}$.

Assim, segundo Fourez e Rouanet ${ }^{23}$, os modelos podem ser comparados a mapas geográficos que não são como cópias de um terreno, mas uma maneira de se localizar; e complementa que o conteúdo de um mapa é determinado, da mesma forma que os modelos, através do projeto que se teve para formulá-los. Dessa forma, pode-se compreender que os modelos teóricos "mapeiam" a estrutura de diferentes domínios.

Ainda segundo Fourez e Rouanet ${ }^{23}$, para ler bem um mapa, é preciso compreender de que modo os símbolos foram determinados. Igualmente, para compreender um modelo científico, será preciso compreender como os conceitos foram construídos. Além disso, existe uma "objetividade" de um mapa no sentido de que, quando se sabe utilizá-lo, ele permite comunicar conhecimentos a respeito do terreno. O que também ocorre com os modelos científicos. $\mathrm{E}$ a possibilidade de utilizá-los no interior de uma comunidade científica que conhece o seu modo de utilização que lhes dá a sua "objetividade", isto é, a sua possibilidade de servir como "objeto" nessa comunidade humana.

Nesse sentido, para Gouveia Júnior ${ }^{24}$, um modelo teórico deve ser passível de mapeamento dentro do espaço teórico onde foi criado e será bem sucedido e melhor considerado caso apresente três condições: (i) Valor de uso (reprodutibilidade): que corresponde ao elevado uso prático; (ii) Valor preditivo: possibilidade de previsão; e (iii) Valor de face: referente à similaridade com o fenômeno proposto.

\section{Reflexões e Visão dos Autores sobre os Modelos Teóricos}

Após reflexões sobre as definições e utilidades dos Modelos Teóricos supracitadas, apresentamos nossa visão sobre como compreendemos os Modelos Teóricos e sua utilidade para a ciência.

É possível compreender que os Modelos Teóricos não são apenas referências ou variáveis que devam ser citadas ou encaixadas em um quadro gráfico ou em uma análise de maneira simples. Cabe ao pesquisador realizar uma grande reflexão sobre o que e como se dará a construção do Modelo Teórico em sua pesquisa, compreendendo seu impacto na vida das pessoas, bem como, identificar e utilizar o método estatístico mais adequado que represente tal modelo.

Do mesmo modo, para que tal pesquisa seja interpretada da melhor maneira, o pesquisador deve dar subsídios teóricos suficientes para que o leitor se aproprie da sua visão de mundo, uma vez que, consideramos que apenas desse modo o pesquisador leitor/avaliador terá total possibilidade de realizar uma verdadeira leitura crítica e/ou avaliação do estudo em questão. Uma vez que, compreendemos que os modelos teóricos de análise são métodos que tentam de forma simplificada, espelhar em seus resultados, o Modelo Teórico hipotéticodedutivo que o pesquisador desenvolveu para a pesquisa, limitando assim, a compreensão do completo raciocínio teórico do pesquisador frente ao tema abordado em seu estudo.

Assim, saber de onde o pesquisador primário partiu e chegou no seu modelo teórico é imprescindível quando pensamos na reprodutibilidade dos estudos. Ou seja, é imprescindível que o pesquisador explicite e represente tais modelos, uma vez que, a reprodutibilidade não depende por completo da qualidade metodológica adotada e sim, da combinação entre 
qualidade metodológica, teórica e de relato dos estudos. É importante ressaltar que de acordo com o nosso posicionamento, toda linha metodológica a ser adotada no estudo, deve ser orientada pelo Modelo Teórico desenvolvido.

Além disso, cabe a reflexão de que apesar de se tratar da reprodutibilidade e uma possível objetividade dos estudos, deve-se compreender que todo estudo por ciência, é subjetivo, uma vez que, ele parte de um modelo teórico hipotético-dedutivo desenvolvido pelo pesquisador.

Assim, definimos Modelos Teóricos como a representação hipotético-dedutiva da vida ou parte dela. Compreendendo assim, como finalidade de todo modelo, conhecer, explicar e/ ou prever a vida ou parte dela em um dado momento do tempo, pessoa e lugar.

Dessa forma, consideramos a Vida como o Modelo Teórico mais complexo que pode existir.

\section{Proposta Teórico-Metodológica para Elaboração de Modelos Teóricos}

Atualmente a elaboração dos modelos teóricos é comumente realizada de duas formas distintas entre os pesquisadores, as quais denominamos:

(i) Elaboração Mecanicista do Modelo Teórico: o pesquisador desenvolve e/ou adota um modelo preexistente sem realizar reflexões sobre sua estrutura e formas conceituais, apenas com o intuito de apresentá-lo, mesmo que de forma limitada e parcial acerca do assunto ou do tema em questão. Durante esse processo, há pouca ou nenhuma interação entre novos conhecimentos e conhecimentos prévios do pesquisador. Trata-se de uma elaboração sem significado e com pouco aprofundamento no tema em questão.

(ii) Elaboração Significativa do Modelo Teórico: o pesquisador desenvolve, adapta e/ou adota um modelo preexistente através de reflexões sobre sua estrutura e formas conceituais por meio de experiências e conhecimentos prévios; assim, atua criando, adaptando e atualizando constantemente tal estrutura a fim de compreender profundamente sobre o assunto ou tema em questão. Durante esse processo, ao contrário da elaboração mecanicista, há muita interação e reflexão entre conhecimentos novos e conhecimentos prévios, compreendendo um modelo teórico com significado, e que servirá para ser reproduzido e testado com maior aprofundamento e qualidade sobre o tema, a fim de rever novas adaptações ao modelo.

Essa classificação foi adaptada a partir do raciocínio do pesquisador e filósofo Ausubel ${ }^{13}$, que desenvolveu a Teoria da Aprendizagem Significativa. Com ela, caracteriza aprendizagem mecânica como aquela em que o sujeito memoriza novos conhecimentos como se fossem informações que podem não Ihe significar nada, porém podem ser reproduzidas a curto prazo e aplicadas automaticamente a situações conhecidas ou necessárias. Durante esse processo, há pouca ou nenhuma interação entre novos conhecimentos e conhecimentos prévios. Trata-se de uma memorização sem significado, mas que serve para ser reproduzida literalmente nas próximas horas ou, talvez, nos próximos dias.

Já a aprendizagem significativa, segundo Ausubel ${ }^{13}$, é definida como a incorporação de novos conhecimentos à estrutura cognitiva com significado, compreensão, capacidade de explicar, transferir e enfrentar situações novas.

Dessa forma, para este estudo, desenvolvemos o que denominamos de abordagem pautada na elaboração significativa do Modelo Teórico, a qual acreditamos ser a forma mais coerente para a elaboração de Modelos Teóricos voltados para pesquisas científicas em Saúde Pública. Para isso, foram realizadas analogias, adaptações e reflexões sobre a literatura no sentido de corroborar com a linha argumentativa sustentada no presente artigo e a qual se almeja levar ao debate público acerca da construção e representação dos Modelos Teóricos.

Além disso, este artigo apresenta um quadro com os principais estudos que foram utilizados como base para o desenvolvimento das etapas que apresentamos para a elaboração dos Modelos Teóricos (Quadro 1). Entretanto, é importante ressaltar que os estudos descritos no quadro abaixo, não apresentam relações diretas entre eles ou mesmo com a forma de criação de modelos teóricos, sendo esta realizada pela compreensão e elucubração deste trabalho, suscitada através de insights e reflexões crítico-científicas.

Nesse sentido, para que a construção do ModeloTeórico se faça através do que denominamos de elaboração significativa, desenvolvemos e explicitamos um passo a passo constituído por sete etapas (Figura 1), com o intuito de auxiliar pesquisadores no desenvolvimento de Modelos Teóricos. 
Quadro 1. Principais Estudos de Fundamentação da Proposta Metodológica.

\begin{tabular}{|l|c|c|}
\hline \multicolumn{1}{|c|}{ Artigos de Fundamentação Teórica } & Autores & Ano \\
\hline Teoria e Realidade & Bunge M. & 1974 \\
\hline A Subsumption Theory of Meaningful Verbal Learning and Retention & Ausubel DP. & 1962 \\
\hline Aquisição e Retenção de Conhecimentos: Uma Perspectiva Cognitiva & Ausubel DP. & 2000 \\
\hline Dual Coding Theory: Retrospect and Current Status & Paivo A. & 1991 \\
\hline What Is the Value of Graphical Displays in Learning & Vekiri I. & 2002 \\
\hline $\begin{array}{l}\text { The Promise of New Ideas and New Technology for Improving Teaching } \\
\text { and Learning }\end{array}$ & Novak JD. & 2003 \\
\hline $\begin{array}{l}\text { Use of Theoretical and Conceptual Frameworks in Qualitative Research } \\
\text { Understanding, Selecting, and Integrating a Theoretical Framework in } \\
\text { Dissertation Research: Creating the Blueprint for Your “House” }\end{array}$ & Green HE. & 2014 \\
\hline
\end{tabular}

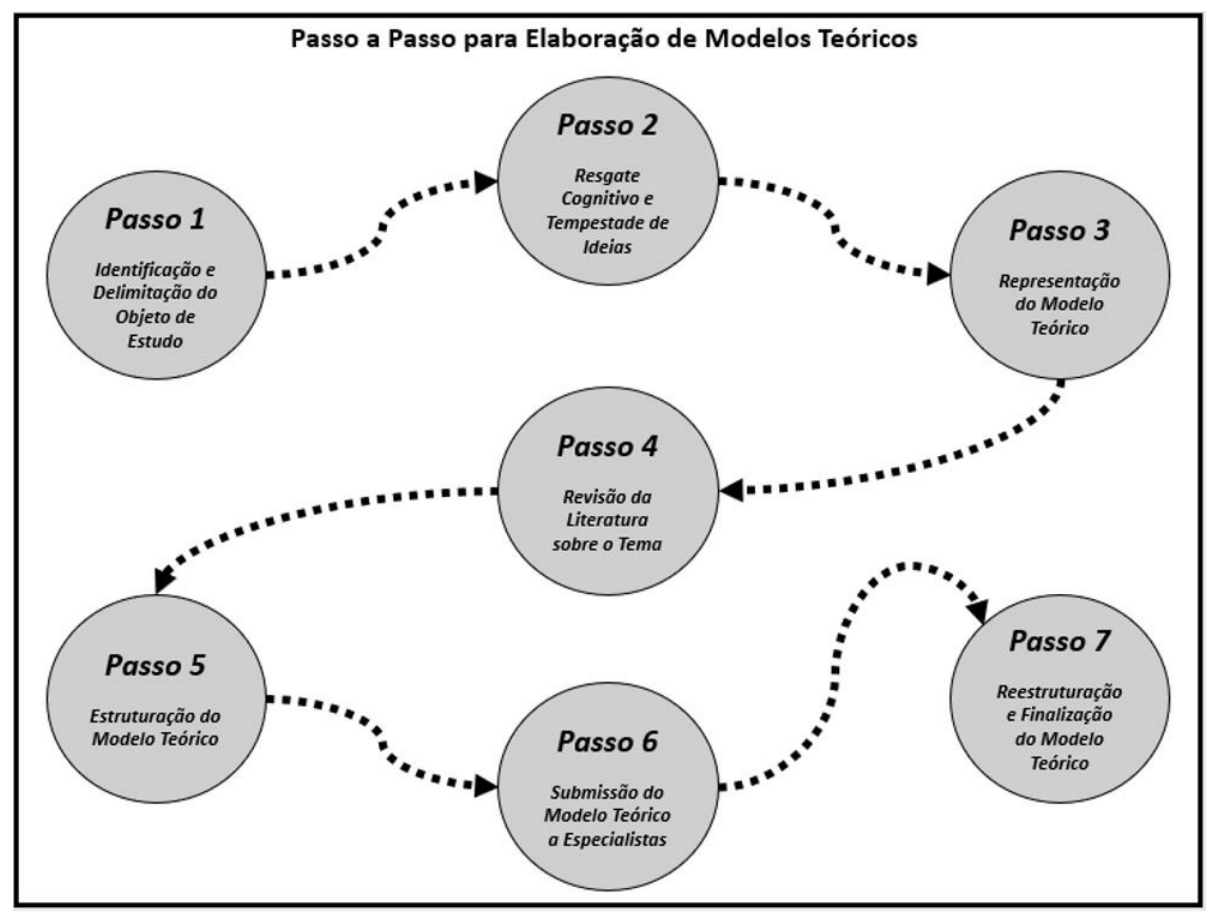

Figura 1. Passo a passo para elaboração de Modelos Teóricos desenvolvido pelos autores.

\section{Passo a Passo para Elaboração de Modelos Teóricos}

\section{PASSO 1 - Identificação e Delimitação do Objeto de Estudo}

Nesta seção, o pesquisador realizará a identificação e delimitação do problema ou inquietação na qual ele pretende se debruçar.

Vale salientar que, o problema de pesquisa ancora todo o estudo e por ele será formada a base a partir da qual se construirá toda a estrutura teórica da pesquisa. Para tanto, faz-se necessário o questionamento sobre se a pergunta a qual se pretende debruçar atende aos critérios específicos relacionados ao seu campo de estudo, uma vez que realizar um estudo, seja qual for a pergunta ou área do conhecimento, requer tempo e, muitas vezes, financiamento, os quais não devem ser desprezados ou negligenciados pela comunidade científica. 
Além disso, após a elaboração da pergunta de pesquisa, inicia-se uma tempestade de ideias e hipóteses para responder tal questão. Nesse momento, o pesquisador lança mão de todo seu repertório cognitivo, dando início à próxima etapa.

\section{PASSO 2 - Resgate Cognitivo e Tempestade de Ideias}

Para esta etapa, acreditamos que a elaboração do modelo teórico deve ser iniciada por experiências prévias do pesquisador sobre a relação causal em questão, nesse sentido é necessário remeter-se novamente, à Teoria da Aprendizagem Significativa de Ausubel ${ }^{13}$.

A Teoria da Aprendizagem Significativa de Ausubel, tem como principal objetivo o aprendizado com significado ${ }^{13,25,26}$. Mas, o autor relata que existem condições para que essa aprendizagem com significado ocorra, sendo elas: a predisposição para aprender; a existência de conhecimentos prévios adequados (conhecidos como subsunçores); e materiais potencialmente significativos.

Para Ausubel ${ }^{13}$, o conhecimento prévio se destaca como a variável que mais influencia a aprendizagem. Do mesmo modo, acreditamos que o conhecimento prévio do pesquisador, é uma variável importante e que deve ser explorada e explicitada na elaboração do Modelo Teórico. Fortalecendo, assim, a compreensão de que são únicos e sua reprodutibilidade por outros cientistas, requererá aprofundamento na visão de mundo do pesquisador primário.

\section{PASSO 3 - Representação do Modelo Teórico}

O Modelo Teórico pode ser representado de diversas formas, sendo a textual a mais usual. Entretanto, sugerimos a sua representação na forma gráfica, uma vez que esta é considerada mais palatável cognitivamente tanto para o pesquisador como leitor, dado que, o processamento mental das imagens causa menor sobrecarga cognitiva que o processamento verbal de um texto ${ }^{27}$.

Allan Paivio ${ }^{28}$ apresenta a Teoria da Dupla Codificação, a qual aponta a existência de dois subsistemas cognitivos interrelacionados: o imagético, concernente à objetos e elementos não verbais; e o verbal, aliado à linguagem. A relação entre os dois sistemas cognitivos faz com que as informações sejam codificadas de modo dual o que facilita a compreensão das relações e conexões estabelecidas na estrutura cognitiva.

Essa potencialidade pode aumentar o número de caminhos que o pesquisador poderá utilizar para resgatar informações referentes ao tema em questão, posto que, um estímulo verbal (ou visual) pode ativar as representações verbal e visual ${ }^{27}$. Dessa forma, sugerimos a utilização e representação dos modelos teóricos, através de mapas conceituais.

Os mapas conceituais são caracterizados como uma estrutura esquemática, que visa representar um conjunto de conceitos inseridos em uma ou mais redes de proposições ${ }^{29}$. Através de seu uso, a informação é apresentada pela forma imagética, pela construção de uma rede verbal, uma vez que a rede é construída pela relação entre conceitos e proposições. Assim, são utilizados dois sistemas cognitivos para sua leitura e interpretação, caminho que facilita a apreensão do conhecimento e o desenvolvimento dos modelos teóricos.

Para construção dos modelos teóricos e sua representação em forma de mapa conceitual, o pesquisador poderá utilizar gratuitamente o software CmapTools (disponível para download em: http://cmap.ihmc.us) ${ }^{30}$.

O CmapTools é uma ferramenta gratuita, desenvolvida pelo Institute for Human Machine Cognition da Universidade do Oeste na Flórida, que permite ao usuário vincular recursos como fotos, imagens, vídeos, gráficos, tabelas, textos, páginas de web ou outros mapas conceituais localizados na internet ou em arquivos pessoais a conceitos ou vincular palavras em um mapa conceitual através de operações simples. Nesse sentido, este software é utilizado para elaborar esquemas conceituais e representá-los graficamente em forma de mapas conceituais, permitindo ao usuário construir, navegar, compartilhar e até mesmo criticar modelos de conhecimento representados como mapas conceituais ${ }^{31,32}$.

O mapa conceitual, portanto, tem o potencial de representar a visão idiossincrática do autor sobre a realidade a qual se debruça a compreender. Suas crenças, conhecimentos 
e experiências prévias, preferências, e modo de olhar e interpretar o mundo influenciam a construção do Modelo Teórico e revelam as particularidades da visão de quem o criou. Essa característica é inerente ao processo de aprendizagem, o uso dos mapas conceituais, entretanto, não se presta apenas a fortalecer o conhecimento individual e único, mas possibilita um caminho metodológico para ampliar as conexões entre conhecimentos prévios e adquiridos, aproximar àqueles desconhecidos e encadeá-los de modo a promover, de fato, a Aprendizagem Significativa, bem como a elaboração significativa do Modelo Teórico.

\section{PASSO 4 - Revisão da Literatura sobre o Tema}

Nesta etapa, o pesquisador dará início a revisão de literatura que, a depender da questão de pesquisa, deverá ser realizada de forma sistemática ou não.

O objetivo principal da revisão é ampliar o conhecimento do pesquisador sobre a forma como seu tema de pesquisa está sendo abordado na literatura, aprimorar e aprofundar seu repertório de conhecimentos e ajustar variáveis, conceitos, dimensões e/ou constructos de seu Modelo Teórico.

O que ocorre entre conhecimentos prévios e conhecimentos novos segundo Ausubel ${ }^{13}$ é uma interação cognitiva, no qual o termo "ancoragem" possui sentido metafórico, dado que, na interação, o "ancoradouro" também se modifica. Dessa forma, Ausubel ${ }^{13}$ relata que o desenvolvimento da Aprendizagem Significativa não é realizado de forma inesperada, pois a apreensão e internalização de significados se apresenta de forma progressiva, e depende da "negociação" entre os significados.

Ausubel $^{13,25}$ relata que tal processo pode ser descrito da seguinte maneira:

1) Um novo conhecimento interage com algum conhecimento prévio, especificamente relevante, no qual o novo conhecimento adquire significado para o aprendiz e o conhecimento prévio adquire novos significados, tornando-se mais elaborado, mais diferenciado, aumentando assim a capacidade de funcionar como subsunçor para outros novos conhecimentos;

2) Durante um certo período, na fase de retenção, o novo conhecimento pode ser reproduzido e utilizado com todas suas características, independente do subsunçor que Ihe gerou significado em um processo denominado de Interação Cognitiva;

3) Simultaneamente, inicia-se um processo de obliteração cujo resultado é um esquecimento (residual) daquele que era um novo conhecimento e que foi aprendido significativamente. Isso quer dizer que aprendizagem significativa não é sinônimo de "nunca esquecer" ou "daquilo que não se esquece";

4) A assimilação obliteradora é a continuidade natural da aprendizagem significativa. Mas, essa obliteração não leva a um esquecimento total. Ao contrário, o novo conhecimento acaba "ficando dentro do subsunçor"e a reaprendizagem é possível e relativamente fácil e rápida.

A partir do raciocínio de Ausubel em sua Teoria da Aprendizagem, acreditamos que a inserção pelo pesquisador de variáveis no modelo teórico se dará da mesma maneira e, assim, através da negociação cognitiva entre variáveis escolhidas por experiências prévias e conhecimentos novos por meio da literatura, sirva de auxilio no processo de elaboração estrutural do modelo, para que este possa ser submetido e avaliado por outros pesquisadores.

\section{PASSO 5 - Estruturação do Modelo Teórico}

Após realização da revisão de literatura, o pesquisador irá confrontar os conhecimentos prévios com os novos conhecimentos, a fim de adequar as variáveis no Modelo, incluindo, mantendo, excluindo e/ou adaptando a sua estrutura.

E, nesse sentido, pode construir uma hierarquia conceitual, iniciando por características mais inclusivas para as mais específicas, tornando clara a diferenciação progressiva, um dos conceitos chaves da teoria de Ausubel. O pesquisador também é estimulado a construir relações de significados entre conceitos aparentemente díspares, tornando clara a reconciliação progressiva - outro conceito chave da teoria de Ausubel. 
Assim, o modelo teórico estruturado em forma de mapa conceitual evidencia-se como um facilitador da meta-aprendizagem, ao possibilitar que o pesquisador adquira a habilidade necessária para construir e adaptar seus próprios conhecimentos.

\section{PASSO 6 - Submissão do Modelo Teórico a Especialistas}

Após estruturação e representação do modelo teórico, é importante que o pesquisador submeta o modelo construído a um grupo de especialistas no tema a ser estudado. A consulta aos especialistas tem por finalidade revisar e discutir possíveis alterações para as variáveis, conceitos, dimensões e/ou constructos contidos no modelo teórico desenvolvido pelo pesquisador e/ou equipe responsável da pesquisa, com o intuito de validar sua estrutura e possibilitar novos arranjos estruturais de acordo com as contribuições de outros profissionais, carregando diferentes visões sobre o tema e apresentando-o de modo mais completo.

Tal processo tem por finalidade determinar a validade de conteúdo e identificar partes do modelo passíveis de modificações, como adições, exclusões ou revisões na sua estrutura ${ }^{33}$.

Esta etapa de submissão é de extrema importância, uma vez que, quando um especialista elabora um modelo teórico em forma de mapa conceitual, ele expõe sua visão madura e aprofundada sobre um tema. Por outro lado, quando um aprendiz constrói o seu mapa conceitual, sua capacidade de perceber características gerais e particulares do tema são exercitadas. Tanto o aprendiz quanto o especialista têm a oportunidade de observar lacunas em seu conhecimento, questionar-se sobre a validade das relações traçadas e seu grau de generalização. Trata-se de um esforço contínuo de aprimoramento da teoria e da técnica, de expansão do conhecimento e raciocínio crítico, além de um exercício de humildade acadêmica no sentido de compreender e aceitar possíveis erros ou inconsistências e buscar formas de aprimorar visões de mundo.

A consulta aos especialistas pode ser realizada de várias maneiras: presencial, semipresencial ou até mesmo à distância. O método mais utilizado é a realização de grupos focais, com reuniões presenciais e/ou a distância, via interação multimídia. Porém, a depender do tema a ser pesquisado, validar o modelo teórico através de grupos focais, pode ser uma tarefa difícil para o pesquisador, tendo em vista dificuldades logísticas, sejam elas temporais ou espaciais.

Nesse sentido, sugerimos o método de submissão do modelo teórico aos especialistas, via e-mail e formulários de autopreenchimento on-line, exemplo Google Docs. (disponível em: https://www.google.com/forms/about/). Este método, além de prescindir da presença física dos profissionais, ainda possibilita a avaliação por meio de cegamento o que, a depender do Modelo e questão de pesquisa elaborados, apresenta-se como um ponto importante, uma vez que a linha argumentativa de um pesquisador pode influenciar a resposta dos demais.

\section{PASSO 7 - Reestruturação e Finalização do Modelo Teórico}

Após avaliação dos especialistas, e como último passo sugerido para a elaboração significativa dos Modelos Teóricos, o pesquisador deve agora realizar as análises finais referente às contribuições e reflexões consensuais, geradas como respostas aos formulários e verificar a necessidade de alteração ou adaptação de variáveis ao modelo.

No caso de, após as análises, o pesquisador decidir modificar o modelo pela inclusão, exclusão ou modificação de variáveis, o modelo deverá igualmente passar pela avaliação dos especialistas para que novamente validem ou não tal modificação.

Por fim, após a realização das etapas sugeridas, o pesquisador e sua equipe, poderão pensar como se dará o desenho de estudo e toda a elaboração das etapas subsequentes necessárias para a elaboração do projeto de pesquisa, embasados no Modelo Teórico desenvolvido.

A fim de demonstrar como os Mapas Conceituais e a ferramenta CmapTools pode contribuir para a visualização, estruturação e adaptação de um Modelo Teórico, visou-se ilustrar de maneira geral, o Passo a Passo desenvolvido para elaboração de Modelos Teóricos (Figura 2). 


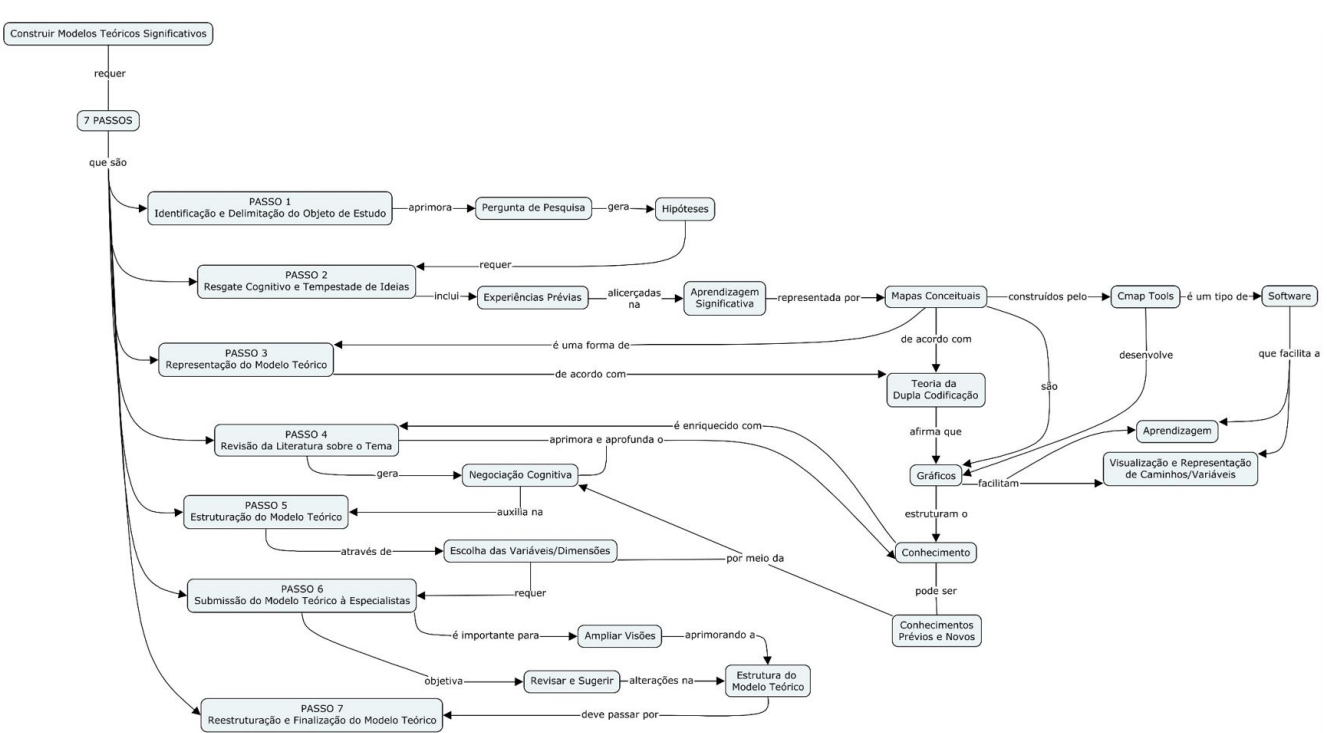

Figura 2. Mapa Conceitual desenvolvido no Cmap Tools para ilustrar o Passo a Passo de elaboração dos Modelos Teóricos.

\section{CONCLUSÃO}

O objetivo do presente artigo foi descrever uma proposta teórico-metodológica para elaboração de modelos teóricos em estudos epidemiológicos na forma de um passo a passo para os pesquisadores. É necessário notar que, tal proposta, não tem pretensão de representarse como a única forma de elaborar modelos, mas de apresentar um método de construí-los o qual o presente estudo demonstra ser coerente e passível de promover a aprendizagem significativa.

Além disso, alterações nas variáveis de um modelo, podem gerar modificações nos resultados de um estudo, supra ou subestimando valores e relações de acordo com a estrutura teórica e conceitual utilizada ${ }^{34}$.

Na Saúde Pública, em particular, os modelos teóricos causais como os Gráficos Acíclicos Direcionados (DAGs), têm apresentado cada vez mais visibilidade e utilidade nos estudos epidemiológicos ${ }^{35}$. Os DAGs, exibem suposições sobre o relacionamento entre variáveis, geralmente chamadas de "nós" no contexto de gráficos. Sua forma de representação teórica conceitual intenta minimizar vieses como confundimento, colisão, entre outros ${ }^{35}$. Entretanto, apesar do seu promissor potencial para os estudos, sua forma de elaboração e escolha das variáveis pelos pesquisadores ainda é um desafio. Dessa forma, acreditamos que a proposta deste artigo pode representar um caminho para a sua elaboração, bem como, de outros formatos de modelos teóricos causais voltados para a Saúde Pública.

Assim, espera-se que este trabalho levante discussões e reflexões sobre os métodos atuais que permeiam o fazer científico e fomentem a utilização e aprofundamento dos Modelos Teóricos como base das pesquisas científicas para a Saúde Pública.

\section{AGRADECIMENTOS}

Agradecemos a pesquisadora sanitarista Érika Fernandes Tritany pela grande contribuição reflexiva e apoio para o andamento e aprimoramento deste manuscrito.

\section{REFERÊNCIAS}

1. Green HE. Use of theoretical and conceptual frameworks in qualitative research. Nurse Res. 2014;21(6):348. http://dx.doi.org/10.7748/nr.21.6.34.e1252. PMid:25059086. 
2. Grant C, Osanloo A. Understanding, selecting, and integrating a theoretical framework in dissertation research: creating the blueprint for your "House". Adm Issues J Educ Pract Res. 2014;4(2):12-26. http:// dx.doi.org/10.5929/2014.4.2.9

3. Cabrera Arana G, Molina Marín G, Rodríguez Tejada C. Base Teórica en una Muestra de Investigaciones de la Facultad Nacional de Salud Pública-Universidad de Antioquia, Colombia 1965-2004. Rev Salud Publica. 2005;7(1):99-111. http://dx.doi.org/10.1590/S0124-00642005000100008. PMid:15906506.

4. Cabrera Arana GA. Uso de teorías y modelos en artículos de una Revista Latinoamericana de Salud Pública, 2000-2004. Rev Saude Publica. 2007;41(6):963-9. http://dx.doi.org/10.1590/S0034-89102007000600011. PMid:18066466.

5. Weed DL. Methods in epidemiology and public health: does practice match theory? J Epidemiol Community Health. 2001;55(2):104-10. http://dx.doi.org/10.1136/jech.55.2.104. PMid:11154249.

6. Luz MT. Notas sobre a política de produtividade em pesquisa no brasil: consequências para a vida acadêmica, a ética no trabalho e a saúde dos trabalhadores. Polit Soc. 2008;7(13):205-28. http://dx.doi. org/10.5007/2175-7984.2008v7n13p205.

7. Borsoi ICF. Trabalho e Produtivismo: Saúde e Modo de Vida de Docentes de Instituições Públicas de Ensino Superior. Cad Psicol Soc Trab. 2012;15(1):81-100. http://dx.doi.org/10.11606/issn.1981-0490.v15i1p81-100.

8. Souza ASR, Silva Junior JR, Agra KF. A política de incentivo e a qualidade da publicação científica no brasil e no mundo. Rev Bras Saúde Mater Infant. 2016;16(1):3-4. http://dx.doi.org/10.1590/180693042016000100001.

9. Santos AS, Perrone CM, Dias ACG. Adaptação à pós-graduação stricto sensu: uma revisão sistemática de literatura. Psico-USF. 2015;20(1):141-52. http://dx.doi.org/10.1590/1413-82712015200113.

10. Almeida Filho N, Costa DC, editors. Teoria epidemiológica hoje: fundamentos, interfaces, tendências. Rio de Janeiro, RJ: Editora Fiocruz: ABRASCO; 1998. http://dx.doi.org/10.7476/9788575412794.

11. Zhang FF, Michaels DC, Mathema B, Kauchali S, Chatterjee A, Ferris DC, et al. Evolution of Epidemiologic Methods and Concepts in Selected Textbooks of the 20 th Century. Soz-PräventivmedizinSocial. Prev Med. 2004;49(2):97-104. http://dx.doi.org/10.1007/s00038-004-3117-8. PMid:15150860.

12. Rother ET. Revisão sistemática x Revisão narrativa. Acta Paul Enferm. 2007;20(2):vi. http://dx.doi. org/10.1590/S0103-21002007000200001.

13. Ausubel DP. A subsumption theory of meaningful verbal learning and retention. J Gen Psychol. 1962;66(2):213-24. http://dx.doi.org/10.1080/00221309.1962.9711837. PMid:13863333.

14. van Ryn M, Heaney CA. What's the use of theory? Health Educ Q. 1992;19(3):315-30. http://dx.doi. org/10.1177/109019819201900304. PMid:1517095.

15. Marx MH, Hillix WA. Sistemas e teorias em psicologia. 2nd ed. São Paulo: Cultrix; 1995.

16. Bunge M. Teoria e realidade. São Paulo: Editora Perspectiva S.A.; 1974.

17. Peruzzo Júnior L, Bunge M. O realismo científico de Mario Bunge. Rev Filos Aurora. 2017;29(46):353. http:// dx.doi.org/10.7213/1980-5934.29.046.ENO1.

18. Parahoo K. Nursing research: principles, process and issues. 2nd ed. Basingstoke: Palgrave Macmillan; 2006.

19. Fain JA. Reading understanding and applying nursing research. 5th ed. Philadelphia: F.A. Davis Company; 2017.

20. Coughlan M, Cronin P, Ryan F. Step-by-step guide to critiquing research. Part 1: Quantitative research. $\mathrm{Br}$ J Nurs. 2007;16(11):658-63. http://dx.doi.org/10.12968/bjon.2007.16.11.23681. PMid:17577184.

21. Almeida Filho N. Epidemiología Sin Números: Una Introducción Crítica a la Ciencia Epidemiológica. Washington, DC: Organizacion Panamericana de la Salud, Oficina sanitaria panamericana, Oficina Regional de la Salud, Organizacion Mundial de la Salud; 1992.

22. Concari SB. Las teorías y modelos en la explicación científica: implicancias para la enseñanza de las ciencias. Ciênc Educ (Bauru). 2001;7(1):85-94. http://dx.doi.org/10.1590/S1516-73132001000100006.

23. Fourez G, Rouanet LP. A construcão das ciências: introducao a filosofia e a ética das ciências. São Paulo: Unesp; 1995.

24. Gouveia A Jr. O conceito de modelo e sua utilização nas ciências do comportamento: breves notas introdutórias. Estud Psicol Camp. 1999;16(1):13-6. http://dx.doi.org/10.1590/S0103-166X1999000100002.

25. Ausubel DP. Aquisição e retenção de conhecimentos: uma perspectiva cognitiva. Lisboa: Paralelo; 2000.

26. Pelizzari A, Kriegl M L, Baron MP, Finck NTL, Dorocinski SI. Teoria da aprendizagem significativa segundo ausubel. Rev PEC. 2005;2:37-42. 
27. Vekiri I. What is the value of graphical displays in learning? Educ Psychol Rev. 2002;14(3):261-312. http:// dx.doi.org/10.1023/A:1016064429161.

28. Paivio A. Dual coding theory: Retrospect and current status. Can J Psychol. 1991;45(3):255-87. http:// dx.doi.org/10.1037/h0084295.

29. Novak JD. The promise of new ideas and new technology for improving teaching and learning. Cell Biol Educ. 2003;2(2):122-32. http://dx.doi.org/10.1187/cbe.02-11-0059. PMid:12888848.

30. Cañas AJ. Cmap Tools Help. cmap.ihmc.us [Internet]. 2004 [cited 2019 Mai 10]. Available from: www.ihmc. us

31. Cañas AJ, Hill G, Carff R, Suri N, Lott J, Gómez G, et al. Cmaptools: A knowledge modeling and sharing environment. In: Concept Maps: Theory, Methodology, Technology, Proceedings of the 1st International Conference on Concept Mapping; 2004 sept; Pamplona, Spain. Pamplona, Spain: Universidad Pública de Navarra; 2004. p. 9.

32. Cañas AJ, Carvajal R, Carff R, Hill G. CmapTools, Web Pages \& Websites. In: Concept Maps: Theory, Methodology, Technology, Proceedings of the 1st International Conference on Concept Mapping; 2004 sept; Pamplona, Spain. Pamplona, Spain: Universidad Pública de Navarra; 2004.

33. Westmoreland D, Wesorick B, Hanson D, Wyngarden K. Consensual Validation of Clinical Practice Model Practice Guidelines. J Nurs Care Qual. 2000;14(4):16-27. http://dx.doi.org/10.1097/00001786-20000700000005. PMid:10881446.

34. Jahn-Eimermacher A, Ingel K, Preussler S, Bayes-Genis A, Binder H. A DAG-based comparison of interventional effect underestimation between composite endpoint and multi-state analysis in cardiovascular trials. BMC Med Res Methodol. 2017;17(1):92. http://dx.doi.org/10.1186/s12874-017-03669. PMid:28676086.

35. Cortes TR, Faerstein E, Struchiner CJ. Utilização de diagramas causais em epidemiologia: um exemplo de aplicação em situação de confusão. Cad Saude Publica. 2016;32(8):e00103115. http://dx.doi. org/10.1590/0102-311X00103115. PMid:27509550. 